\title{
APPROXIMATE THIRD INTEGRALS IN AXISYMMETRIC GALAXIES AND THE TILT OF THE VELOCITY ELLIPSOID IN THE SOLAR NEIGHBORHOOD
}

\author{
STEPHEN M. KENT ${ }^{1}$ \\ Harvard-Smithsonian Center for Astrophysics, Mail Stop 20, 60 Garden Street, Cambridge, Massachusetts 02138
}

TIM DE ZEEUW

Sterrewacht Leiden, Postbus 9513, 2300 RA Leiden, The Netherlands

Received 3 October 1990; revised 12 August 1991

\begin{abstract}
We present three different methods for calculating approximate third integrals of the motion for orbits in axisymmetric potentials. All three methods are based on approximating the orbits by motion in a separable potential. The results are applied to the Carlberg-Innanen potential for the Galaxy and allow a determination of the tilt of the velocity ellipsoid in the solar neighborhood.
\end{abstract}

\section{INTRODUCTION}

The motion of a particle in an arbitrary potential $\Phi$ is constrained in six-dimensional phase space by the isolating integrals of the motion, which are constant along an orbit (Jeans 1915). If the potential is axisymmetric, two integrals are the total energy $E$ and the angular momentum about the symmetry axis $h$. The existence of such a third, nonclassical isolating integral depends on the form of the potential. Axisymmetric Stäckel potentials (Stäckel 1890; Eddington 1915; Lynden-Bell 1962; de Zeeuw 1985) lead to a separation of the Hamilton-Jacobi equation in spheroidal coordinates and are the most general class of axisymmetric galaxy potentials known with exact isolating third integrals. Many other special two-dimensional potentials also have exact nonclassical isolating integrals (Hietarinta 1987). However, most galaxy potentials do not fall into these categories, and one must normally resort to numerical orbit integrations to find out whether a third integral exists. Typically one finds that galactic potentials give rise to both regular orbits that possess isolating third integrals, and stochastic orbits that do not (Ollongren 1962; Martinet 1974).

A distribution of stars in a potential with three isolating integrals can have a velocity ellipsoid with three unequal principal axes. It is well known that this is indeed the case for virtually all types of stars in the solar neighborhood (e.g., Oort 1928; Freeman 1987), strongly suggesting that the stellar orbits in the Galaxy possess a third isolating integral. In general, the shape and orientation of the velocity ellipsoid for an ensemble of stars depends on the details of both the potential and the distribution function of stars. A classic problem in Galactic dynamics is calculating the tilt of the velocity ellipsoid for stars above the Galactic plane. This quantity enters into the equations of hydrostatic equilibrium and is needed to calculate the asymmetric drift of stars (Binney \& Tremaine 1987) and the surface mass density (Kuijken \& Gilmore 1989a,b; Statler 1989) in the solar neighborhood. In the latter case, the uncertainty in the tilt term leads to a $20 \%$ variation in the inferred mass density and formally is the largest single source of error. Although the tilt term is often regarded as being unknown, we show that it can be estimated to good accuracy even without perfect knowledge of the Galactic potential or the distribution function of stars.

To calculate the orientation and shape of the velocity el-

${ }^{1}$ Current address: Fermilab, MS 127, P. O. Box 500, Batavia, IL 60510. lipsoid in the solar neighborhood, it is important to have analytic or numerical approximations to the third integral. Following the pioneering work of Contopoulos (1960), many perturbation expansions have been constructed for a third integral in potentials that deviate only slightly from cases with exact third integrals (e.g., van de Hulst 1962; Saaf 1968; Manabe 1979; Verhulst 1979). Manabe (1979) has compared the accuracy of several of these approximations for stars orbiting in the solar neighborhood. He finds that even the best approximations predict orbit shapes that deviate noticeably from the true shape for orbits that have an extent of only 2-3 kpc. These approximations are not adequate for calculating the tilt term because typical disk stars in the solar neighborhood do reach to this height above the Galactic plane.

This paper presents three methods (two of which are new) for finding approximate third integrals in axisymmetric potentials applicable to the major family of short-axis tube orbits. All methods approximate an orbit by motion in a separable potential. Each method has its own advantages for different types of orbits. We find that we can often approximate orbits to much higher accuracy than is possible with previous perturbation-type methods. In particular, our methods allow an accurate calculation of the velocity ellipsoid in the solar neighborhood.

\section{FITTING ORBITS}

The three-dimensional motion in the gravitational potential $\Phi$ of an axisymmetric galaxy is conveniently described as two-dimensional motion in a meridional plane, with Hamiltonian

$$
H=\frac{1}{2}\left(p_{R}^{2}+p_{z}^{2}\right)+V(R, z),
$$

where $R$ is the radial coordinate, $z$ is the vertical coordinate, $p_{R}$ and $p_{z}$ are the linear momenta, and $V(R, z)$ is the reduced, or effective, potential, defined as

$$
V(R, z)=\Phi(R, z)+\left(h^{2} / 2 R^{2}\right) .
$$

Here $h$ is the angular momentum component about the symmetry axis. We assume that $V$ is symmetric in $z$.

The most general axisymmetric potential that gives rise to three isolating integrals is a Stäckel potential (Kuzmin 1956; Hori 1962). Orbits in these potentials generally fill donutshaped three-dimensional volumes which are bounded by coordinate surfaces, and hence the orbits are nowadays re- 
ferred to as short-axis tubes. When the potential is not of Stäckel form, we cannot write down exact analytic third integrals. However, several schemes for computing numerical approximations exist. Here we examine three methods for computing approximate third integrals which are of use for describing orbits of stars in the solar neighborhood. Each method has its own domain of applicability.

\subsection{Local Fitting}

In a scheme developed originally by van de Hulst (1962), it is possible to fit potentials to fourth order in both the $R$ and $z$ directions simultaneously by using Stäckel potentials.

The Hamiltonian for the special oblate potentials $V(R, z)$ that are of Stäckel form separates in prolate spheroidal coordinates $(u, \phi, v)$, where $u$ and $v$ are two-dimensional elliptic coordinates in a meridional plane of constant $\phi$, defined by the conformal transformation $R+i z=\Delta \sinh (u+i v)$, so that

$$
R=\Delta \sinh u \cos v ; \quad z=\Delta \cosh u \sin v,
$$

where $0 \leqslant u<\infty,-\pi / 2 \leqslant v \leqslant \pi / 2$. The foci lie along the $z$ axis, at $z= \pm \Delta$. The momenta $p_{u}$ and $p_{v}$ are related to $p_{R}$ and $p_{z}$ by

$$
\begin{aligned}
& p_{u}=\Delta\left(p_{R} \cosh u \cos v+p_{z} \sinh u \sin v\right), \\
& p_{v}=\Delta\left(p_{z} \cosh u \cos v-p_{R} \sinh u \sin v\right) .
\end{aligned}
$$

The gravitational potential $\Phi$ is of Stäckel form if it can be written as

$$
\Phi(u, v)=\frac{F^{*}(u)+G^{*}(v)}{\Delta^{2}\left(\cosh ^{2} u-\sin ^{2} v\right)} .
$$

The effective potential is

$$
V=\Phi(u, v)+\frac{h^{2}\left(\sec ^{2} v+\operatorname{csch}^{2} u\right)}{2 \Delta^{2}\left(\cosh ^{2} u-\sin ^{2} v\right)},
$$

so that $V$ is of Stäckel form if $\Phi$ is.

In a potential of this kind, all orbits are short axis tubes and have an exact third integral $I_{3}$ given by

$$
\begin{aligned}
I_{3} & =\Delta^{2} E \cosh ^{2} u-\frac{1}{2} p_{u}^{2}-F^{*}(u)-\frac{h^{2}}{2 \sinh ^{2} u} \\
& =\Delta^{2} E \sin ^{2} v+\frac{1}{2} p_{v}^{2}+G^{*}(v)+\frac{h^{2}}{2 \cos ^{2} v}
\end{aligned}
$$

Near a circular orbit at $R=R_{c}$ in the equatorial plane, the effective potential can be expanded in powers of $x=R-R_{c}$ and $z$ as follows:

$$
V(x, z)=\sum_{k=0}^{\infty} \sum_{l=0}^{\infty} V_{k l} x^{k} z^{l},
$$

where $V_{10}=0$, and only even values of $l$ occur, by symmetry. The question arises how well this expansion can be matched by a similar one for a separable potential. This problem was solved by van de Hulst (1962), in his investigation of epicyclic motion in the meridional plane of an axisymmetric potential. He showed that, by expanding a separable $V$ around a point on the $x$ axis that does not coincide with the origin, one can always fit the expansion (8) up to and including quartic terms. At quintic order one can fit the coefficients $V_{50}$, but the values of $V_{32}$ and $V_{14}$ are then fixed. Similar constraints occur at all higher orders.

In this local fitting, the $(u, v)$-coordinate system has to be chosen such that its origin lies at $\left(-x_{0}, 0\right)$, with

$$
x_{0}=\frac{4 V_{12}\left(V_{20}-V_{02}\right)}{2 V_{22}\left(V_{20}-4 V_{02}\right)-3 V_{12}\left(V_{30}-2 V_{12}\right)},
$$

and the semi-focal distance $\Delta$ must be taken as

$$
\Delta^{2}=-x_{0}^{2}+x_{0}\left[\left(V_{20}-4 V_{02}\right) / V_{12}\right] \text {. }
$$

As a result, the boundaries of the area in the meridional plane filled by the orbit are elliptic coordinate lines, i.e., pieces of confocal ellipses and hyperbolae.

The third integral of the best fitting Stäckel potential may be written explicitly in terms of the original expansion coefficients $V_{k l}$ themselves. The general result (de Zeeuw 1984) is

$$
I_{3}=\frac{1}{2}\left(x p_{z}-z p_{R}\right)^{2}+\frac{1}{2} \Delta^{2}\left[p_{z}^{2}+W(x, z)\right],
$$

where

$$
\begin{aligned}
W(x, z)= & \frac{\left(x_{0}^{2}+\Delta^{2}\right)}{\Delta^{2}}\left[V_{02} z^{2}-\frac{2 V_{12} V_{02}}{\left(V_{20}-4 V_{02}\right)} x z^{2}\right. \\
& +\left(\frac{1}{2} V_{22}+\frac{V_{12}\left(2 V_{12}-3 V_{30}\right)}{4\left(V_{20}-4 V_{02}\right)}\right) x^{2} z^{2} \\
& \left.+\left(V_{04}-\frac{V_{12}^{2}}{4\left(V_{20}-4 V_{02}\right)}\right) z^{4}+\cdots\right]
\end{aligned}
$$

Two cases deserve special attention. If $V_{02}=V_{20}$, then we find from Eq. (9) that $x_{0}=0$ and $\Delta=0$, so that the potential is fitted with a function of $x^{2}+z^{2}$. For $V_{20}=4 V_{02}$ the point $\left(x_{0}, 0\right)$ coincides with a focus of the elliptic coordinates $(u, v)$, in the neighborhood of which the coordinates are approximately parabolic. This case is not relevant to the motion in the solar neighborhood, where $V_{02}>V_{20}$.

\subsection{Least-Squares Fitting}

The local fitting procedure predicts that all orbits of a given $h$ are bounded by the same spheroidal coordinate system. For orbits of sufficiently high energy, the local fitting approximation of Sec. 2.1 no longer works well. However, we find that in many cases orbits can still be approximated by motion in a Stäckel potential provided that the potential is chosen to be a good "average" approximation to the true potential in the region covered by a particular orbit. We now develop a practical method to find the appropriate spheroidal coordinate system and the resulting third integral approximation tailored for individual orbits.

By Eq. (7), the following function should vanish along a given orbit:

$$
Q=I_{3}+\frac{1}{2} p_{u}^{2}+F^{*}(u)+\frac{h^{2}}{2 \sinh ^{2} u}-\Delta^{2} E \cosh ^{2} u .
$$

For a given choice of coordinates, the function $F^{*}(u)$ is most easily found by evaluating Eq. (5) along the $R$ axis,

$$
F^{*}(u)=\Delta^{2} \Phi(u, 0) \cosh ^{2} u \text {. }
$$

If the potential is not separable, then the function given by Eq. (13) will not be constant along an orbit. However, having integrated a particular orbit numerically, we can adjust the parameters $\Delta$ and $x_{0}$ of the coordinate system so as to make that function as nearly constant as possible. This is the basis of our fitting scheme. Given a numerically integrated orbit, we adjust $\Delta, x_{0}$, and the as-yet unknown $I_{3}$ so as to minimize the rms variations in Eq. (13). Specifically, we minimize the quantity $\int Q^{2} d t$ along an orbit. The orbit is 
integrated for a time long enough to define its boundaries. By fitting the coordinate system to a specific orbit rather than, say, fitting a Stäckel approximation to the true potential over some region (as was done by de Zeeuw \& Lynden-Bell 1985), we are able to tailor the third integral as closely as possible to that orbit, and we also allow for the fact that in general, different orbits are best fit by different approximations.

\subsection{Conformal Fitting}

Finally, we look at orbits that have an arbitrarily large extent in the $R$ direction but that deviate only slightly in the $z$ direction. These orbits are typical of Galactic disk stars where the $R$ dispersion is much larger than the $z$ dispersion. We can think of these orbits as oscillating slightly about the periodic orbit that lies in the $R$ axis. ${ }^{2}$

We introduce a new coordinate system $(u, v)$ by a conformal transformation:

$$
R+i z=f(u+i v) \text {. }
$$

Let the $R$ axis coincide with the $u$ axis for $z=v=0$. Because the transformation is conformal, one has $\partial R / \partial u=\partial z / \partial v$ and $\partial R / \partial v=-\partial z / \partial u$. In terms of the new momenta, the kinetic energy is $\frac{1}{2}\left(p_{R}^{2}+p_{z}^{2}\right)=\frac{1}{2} J\left(p_{u}^{2}+p_{v}^{2}\right)$, where $J$ is the Jacobian of the transformation: $J=\left[(\partial R / \partial u)^{2}+(\partial z / \partial u)^{2}\right]^{-1}$. The equation $H=E$ can then be written in the form

$$
\frac{1}{2}\left(p_{u}^{2}+p_{v}^{2}\right)+\frac{U(u, v)}{J}=0,
$$

where $U=V-E$.

In Hamilton-Jacobi theory, the Hamiltonian is separable if $U / J$ can be written in the form $U / J=F(u)+G(v)$. In that case, Eq. (16) splits into two parts, each of which is constant,

$$
\begin{aligned}
& \frac{1}{2} p_{u}^{2}+F(u)=-I_{3}, \\
& \frac{1}{2} p_{v}^{2}+G(v)=I_{3} .
\end{aligned}
$$

Here the signs are chosen so that if $G(0)=0$, then $I_{3}>0$. Global separation for axisymmetric models occurs only for Stäckel potentials, as we have discussed already. However, our aim here is to find a coordinate system such that Eq. (1) is separable to just second order in $v$, and that can be done for a much larger class of potentials. First, Eq. (15) can be expanded for small $v$,

$$
\begin{aligned}
& R=f-\frac{1}{2} v^{2} f^{\prime \prime}, \\
& z=v f^{\prime}-\frac{1}{6} v^{3} f^{\prime \prime \prime},
\end{aligned}
$$

where $f$ is now a real function of $u$, and the primes indicate differentiation with respect to $u$. Then, to order $v^{2}, J$ is given by

$$
\frac{1}{J}=f^{\prime 2}+\left(f^{\prime \prime 2}-f^{\prime} f^{\prime \prime \prime}\right) v^{2}
$$

Next, the function $U$ must be expanded for small $v$,

$$
U=U(u, 0)+\frac{1}{2}\left(\frac{\partial^{2} U}{\partial v^{2}}\right)_{v=0} v^{2} .
$$

The second derivative can be written in a more useful form as follows:

${ }^{2}$ Note that in three dimensions this "periodic" orbit fills an annulus in the
equatorial plane.

$$
\begin{aligned}
& \frac{\partial U}{\partial v}=\frac{\partial U}{\partial R} \frac{\partial R}{\partial v}+\frac{\partial U}{\partial z} \frac{\partial z}{\partial v}=-f^{\prime \prime} \frac{\partial U}{\partial R} v+f^{\prime} \frac{\partial U}{\partial z}, \\
& \frac{\partial^{2} U}{\partial v^{2}}=-f^{\prime \prime} \frac{\partial U}{\partial R}+f^{\prime 2} \frac{\partial^{2} U}{\partial z^{2}}+\cdots,
\end{aligned}
$$

where the dots indicate terms that vanish when evaluated at $v=0$. Combining Eqs. (19), (20), and (21) yields

$$
\begin{aligned}
\frac{U}{J}= & f^{\prime 2} U(u, 0)+\left[-\frac{1}{2} f^{\prime 2}\left(f^{\prime \prime} \frac{\partial U}{\partial R}+f^{\prime 2} \frac{\partial^{2} U}{\partial z^{2}}\right)\right. \\
& \left.+\left(f^{\prime \prime 2}-f^{\prime} f^{\prime \prime \prime}\right) U\right] v^{2}=0 .
\end{aligned}
$$

Again, $U$ and its derivatives are to be evaluated at $v=0$.

Equation (16) is separable to second order in $v$ only if the quantity in brackets in Eq. (22) is a constant:

$$
\left(f^{\prime \prime 2}-f^{\prime} f^{\prime \prime \prime}\right) U+\frac{1}{2} f^{\prime 2}\left(-f^{\prime \prime} \frac{\partial U}{\partial R}+f^{\prime 2} \frac{\partial^{2} U}{\partial z^{2}}\right)=K \text {. }
$$

Equation (23) is the desired result. It is a nonlinear third order equation for $R=f(u)$. The function $U$ and its various derivatives are known functions of $R$ (and hence of $f$ ) and are evaluated at $z=0$. A solution to Eq. (23) requires that we specify three integration constants plus the as-yet unknown separation constant $K$. The integration constants may be chosen to be the values of $f^{\prime}=a$ and $f^{\prime \prime}=b$ at some point and the value of $u=u_{0}$ at that point. Two of these constants, $a$ and $u_{0}$, are arbitrary and simply correspond to a linear rescaling and shift in origin of the $(u, v)$ coordinate system. The remaining two constants may be obtained by recognizing that Eq. (23) has two singular points where $U=0$ (corresponding to the turning points of the periodic orbit), and so $f^{\prime \prime}$ cannot be specified arbitrarily at these points, but rather must satisfy

$$
f^{\prime \prime}=\frac{-\left(2 K / f^{\prime 2}\right)+f^{\prime 2}\left(\partial^{2} U / \partial z^{2}\right)}{\partial U / \partial R} .
$$

Since there are two singular points where Eq. (23) must be satisfied, both $b$ and $K$ are determined uniquely.

From a computational standpoint, Eq. (23) is most conveniently integrated using the shoot-to-midpoint method (Press et al. 1986). At the lower singular point, one selects $u_{-}=0, f_{-}^{\prime}=a=1$, and makes a guess at $K$; at the upper endpoint one makes a guess at $f^{\prime}{ }_{+}$and $u_{+}$. Equation (23) is then integrated from each singular point to a midpoint $f=R_{m}$. At the singular points, the starting value of $f^{\prime \prime \prime}$ is given by

$$
\begin{aligned}
f^{\prime \prime \prime}= & \frac{2 f^{\prime}}{3(\partial U / \partial R)}\left(-\frac{1}{2} f^{\prime \prime} \frac{\partial^{2} U}{\partial R^{2}}+2 f^{\prime \prime} \frac{\partial^{2} U}{\partial z^{2}}\right. \\
& \left.+\frac{1}{2} f^{\prime 2} \frac{\partial^{3} U}{\partial R \partial z^{2}}\right) .
\end{aligned}
$$

The values of $K, f^{\prime}{ }_{+}$, and $u_{+}$are adjusted iteratively so as to make $f^{\prime}, f^{\prime \prime}$, and $u$ continuous at $R_{m}$.

The third integral is given to second order by

$$
I_{3}=-\left[\frac{1}{2} p_{u}^{2}+f^{\prime 2} U(u, 0)\right]=\frac{1}{2} p_{v}^{2}+K v^{2} \text {. }
$$

If $K>0$, the periodic orbit about which we are expanding is stable, else it is unstable. The boundaries of the stable orbits are given by $I_{3}=-f^{\prime 2} U(u, 0)$ and $v= \pm \sqrt{I_{3} / K}$.

For potentials that are separable in Cartesian coordinates, $f=1$ everywhere, and $I_{3}$ reduces to the energy $E_{z}$ in the $z$ direction. For spherically symmetric potentials, the solution 
to Eq. (23) is $f=a \exp \left(u-u_{0}\right)$, and $I_{3}$ reduces to $\frac{1}{2}\left(L^{2}-h^{2}\right)$ where $L$ is the total angular momentum of the orbit. Note that the transformation function $f$ depends on the energy $E$ and angular momentum $h$ of an orbit (via their appearance in the function $U$ ) but not on $I_{3}$.

Although the above analysis is only valid to second order in $v$, i.e., for small $v$, numerical integration of orbits in test potentials shows that the coordinate system described by the function $f$ sometimes provides a good description of the orbits with substantial $v$ amplitudes. In such cases the third integral in Eq. (26) can be written as

$$
I_{3}=\frac{1}{2} p_{v}^{2}+G(v),
$$

where $G(v)$ is the function $U / J$ evaluated along any curve $u=$ constant. The upper and lower boundaries of an orbit are then found by solving this equation when $p_{v}=0$.

For comparison with the local fitting of Sec. 2.1, we note that the function $f$ of the transformation to the specific $(u, v)$ coordinate system used in the local fitting is given by

$$
f(u+i v)=R+i z=\Delta \sinh (u+i v)+R_{c}-x_{0}
$$

and the Jacobian is

$$
\frac{1}{J}=\Delta^{2}\left(\cosh ^{2} u-\sin ^{2} v\right) \text {. }
$$

The function $U$ becomes

$$
\begin{aligned}
U= & \frac{F^{*}(u)+G^{*}(0)}{\Delta^{2} \cosh ^{2} u}-E+\frac{h^{2}}{2 \Delta^{2} \sinh ^{2} u}+\frac{1}{\Delta^{2}} \\
& \times\left(\frac{F^{*}(u)+G^{*}(0)}{\cosh ^{4} u}+\frac{G^{* \prime \prime}(0)}{2 \cosh ^{2} u}+\frac{h^{2}}{2 \sinh ^{2} u}\right) v^{2} .
\end{aligned}
$$

Equation (23) reduces to

$$
\Delta^{2} E+\frac{1}{2} G^{* \prime \prime}(0)+\left(h_{2} / 2\right)=K \text {. }
$$

We remark that a Stäckel potential has only one arbitrary function $F^{*}(u)$ plus two constants $\left[\Delta\right.$ and $\left.G^{* \prime \prime}(0)\right]$ that can be specified along $z=v=0$. Equation (23) allows one to specify two independent arbitrary functions $\left(U\right.$ and $\left.\partial^{2} U / \partial z^{2}\right)$. Hence the conformal fitting method allows one to find approximate third integrals for potentials that are more general than any Stäckel potential. However, it is valid only to second order in $v$, whereas the local fitting method works through fourth order.

\subsection{Examples}

Figure 1 shows an application of these fitting methods to four orbits. The first three are orbits of stars in the Carlberg \& Innanen (1987) model for the gravitational field of the Galaxy. The orbits were integrated numerically by launching a particle from $R=8.5 \mathrm{kpc}, z=0$ with the indicated velocity. For each orbit we have computed an appropriate prolate spheroidal coordinate system using both the local fitting approximation and the more general least-squares fitting method. Given a coordinate system, we then approximate the potential using Eq. (5) with $F^{*}$ and $G^{*}$ given by $F^{*}(u)=\Delta^{2}\left(\cosh ^{2} u\right) \Phi(u, 0)$ and $G^{*}(v)=\Delta^{2}\left(\cosh ^{2} u_{L}\right.$ - $\left.\sin ^{2} v\right) \Phi\left(u_{L}, v\right)$, where $u_{L}$ is the value of $u$ corresponding to the launch radius of the stars $(8.5 \mathrm{kpc})$. The boundaries of the orbits are then found by solving Eqs. (7) for the values of $u$ and $v$ where $p_{u}=p_{v}=0$. The boundaries are plotted in the figure. For the first orbit, both methods appear to fit the orbit boundary well. However, the least-squares fit actually does
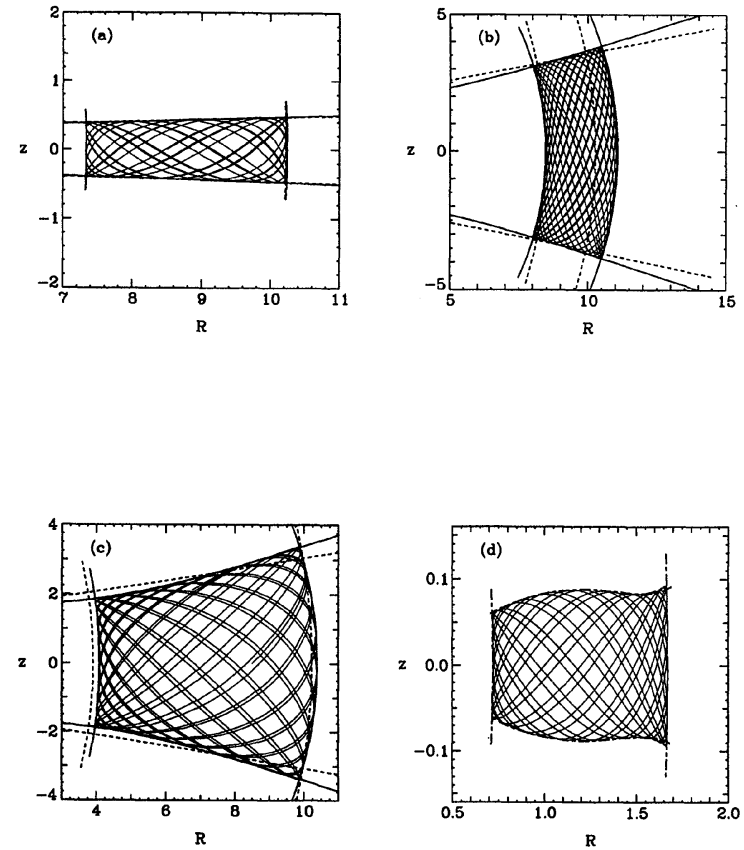

FIG. 1. Meridional plane diagrams for four orbits with boundaries calculated by three different methods. Orbits in (a)-(c) were run in the Carlberg-Innanen model for the Galaxy. All orbits were launched from $R=8.5 \mathrm{kpc}$ with $\left(v_{R}, v_{\phi}, v_{z}\right)$ velocities of $(50,0,30)$ and $(10,0,120)$, and $(100,-80,100) \mathrm{km} \mathrm{s}^{-1}$, respectively. Orbit in (d) was run in a potential described in the text. The orbit was launched from $R=1, z=0$ with velocities $(0.4,0,0.1)$. Note that the vertical scale in (d) has been stretched. Boundaries were computed using the conformal fitting method (long- and short-dashed lines), local fitting method (short-dashed lines), and least-squares fitting method (heavy solid lines).

significantly better. The rms error in the predicted velocity along the $u$ axis, for example, is about $14 \mathrm{~km} \mathrm{~s}^{-1}$ for the localfitting approximation but is essentially 0 for the leastsquares approximation. For the second and third orbits, which travel to nearly $4 \mathrm{kpc}$ above the galactic plane, the local-fitting approximation now fails quite badly, whereas the least-squares approximation still provides an excellent fit: the rms error in the $u$ velocity is still only $1-3 \mathrm{~km} \mathrm{~s}^{-1}$ for the latter.

Although lines for the conformal fitting method are not plotted in Figs. 1(a)-1(c), it gives virtually identical results with the local fitting method.

Figure 1(d) shows an orbit integrated in the potential $\Phi=-1 / \sqrt{R^{2}+z^{2}}-0.1 \cos (3 z)$. This potential is not chosen to match any particular galaxy model, although the cosine portion might be thought of as representing a perturbation due to rings. Although the orbit oscillates only slightly in the $z$ direction, the top and bottom boundaries of this orbit are too convoluted to be represented by spheroidal coordinates. As shown in the figure, the coordinate system generated by the conformal fitting method works much better.

To summarize, many orbits in smooth potentials can be well approximated by motion in a separable potential, but the best spheroidal coordinate system and approximating potential differ for different orbits. Consequently, the least- 
squares fitting method gives sufficiently better results than the other methods that it is preferred in general. There are two situations in which this is not the case. First, if the orbit has low amplitude in both the $R$ and $z$ directions, then the least-squares method becomes unstable. In this case the local fitting method gives an adequate approximation. Second, if the potential is sufficiently complicated, then the orbits cannot be described by spheroidal coordinates. In this case the conformal fitting method gives a superior approximation, although it works well only for orbits of small amplitude in the $z$ direction.

\section{THE TILT OF THE VELOCITY ELLIPSOID}

In a separable potential, the axes of the velocity ellipsoid for a distribution of stars are aligned with the coordinate system in which the Hamilton-Jacobi equation separates (Eddington 1915). Thus, for example, if the potential is spherically symmetric, one axis of the ellipsoid will point toward the galaxy center. In a nonseparable potential, however, each regular orbit has its own distinct principal axes, and the orientation of the velocity ellipsoid for an ensemble of stars depends on the detailed distribution function of the stars.

The tilt of the velocity ellipsoid enters into the equations of hydrostatic equilibrium in cylindrical coordinates (Binney \& Tremaine 1987); e.g., the $z$ equation has the form

$$
v \frac{\partial \Phi}{\partial z}=\frac{\partial}{\partial z}\left(v \sigma_{z z}^{2}\right)+\frac{1}{R} \frac{\partial}{\partial R}\left(R v \sigma_{R z}^{2}\right),
$$

where $v$ is the space density of stars and $\sigma_{i j}^{2}$ are the various second moments of the velocity distribution function. The second term on the right side of this equation involves the tilting of the velocity ellipsoid. Kuijken \& Gilmore $(1989 a, b)$ have shown that this term contributes an uncertainty of $20 \%$ in dynamical estimates of the surface mass density of the galaxy in the solar neighborhood.

The magnitude of the tilting in the solar neighborhood can be estimated as follows. If $V_{R}$ and $V_{z}$ are the $R$ and $z$ velocities of a star at some point, then the tilting term is defined to be $\sigma_{R z}^{2}=\left\langle V_{R} V_{z}\right\rangle$, where \langle\rangle denotes the density-weighted average over all orbits at a fixed point, and we have assumed that there is no net streaming motion in either the $R$ or $z$ directions. For a single orbit, a star can pass through a given point in four possible directions. The contribution of this orbit to $\sigma_{R z}^{2}$ can be found by transforming to the best-fitting $(u, v)$ coordinate system for that orbit as found by the methods of Sec. 2. Let $V_{R}=V_{u} \cos \theta-V_{v} \sin \theta$ and $V_{z}=V_{u} \sin \theta+V_{v} \cos \theta$, where $\theta$ is the slope of a line of constant $v$. Then since $\left\langle V_{u} V_{v}\right\rangle=0$ (a star can have velocities $\pm V_{u}$ and $\left.\pm V_{v}\right)$, we have $\left\langle V_{R} V_{z}\right\rangle=\left(V_{u}^{2}-V_{v}^{2}\right)$ $\times \cos \theta \sin \theta$. In the solar neighborhood, where $\theta$ is small for all orbits, we have $V_{u}^{2} \approx V_{R}^{2}$ and $V_{v}^{2} \approx V_{z}^{2}$, and hence the tilting term $\sigma_{R z}^{2}$ for a single orbit can be approximated as

$$
\sigma_{R z}^{2} \approx\left(V_{R}^{2}-V_{Z}^{2}\right) \theta
$$

$\theta$ is most easily calculated using the conformal function $f$ of Sec. 2.3: along a line of constant $v$,

$$
d R=(\partial R / \partial u) d u, \quad d z=(\partial z / \partial u) d u,
$$

SO

$$
\theta=\frac{d z}{d R}=\frac{\partial z / \partial u}{\partial R / \partial u}=\frac{f^{\prime \prime} z}{f^{\prime 2}}
$$

with $f$ and its derivatives are evaluated at $(R, 0)$. Hence the tilting term is

$$
\sigma_{R z}^{2} \approx\left(V_{R}^{2}-V_{z}^{2}\right) \frac{f^{\prime \prime} z}{f^{\prime 2}}
$$

The magnitude of this term is often expressed in terms of the corresponding value that it would have if the velocity ellipsoid pointed towards the galactic center; in that case, $\theta \approx z / R=z / f$. The correction factor is therefore

$$
C=\frac{f f^{\prime \prime}}{f^{\prime 2}}
$$

For the specific case of a local Stäckel fit in spheroidal coordinates centered at $R=R_{c}-x_{0}, z=0$, as described in Sec. 2.2, we have [from Eq. (28)]

$$
C=\frac{R\left(R-R_{c}+x_{0}\right)}{\Delta^{2}+\left(R-R_{c}+x_{0}\right)^{2}} .
$$

The above derivation of $C$ is valid for a single orbit, and in general we expect each orbit to have a different value of $C$. For an ensemble of orbits we define an average $\langle C\rangle$ such that

$$
v \sigma_{R z}^{2}=\langle C\rangle v\left(\sigma_{R R}^{2}-\sigma_{z z}^{2}\right) z / R \text {. }
$$

We have evaluated the factor $C$ for a variety of orbits characteristic of the kinematics of old disk stars in the solar neighborhood using the Carlberg \& Innanen (1987) model for the gravitational field of the Galaxy but with scale parameters taken from Kuijken \& Gilmore (1989a). We have calculated the tilt term by numerically integrating the orbits and approximating their motion where possible using the least-squares method described in Sec. 2.2. For orbits with very small excursions in both $R$ and $z$, the least-squares method is unstable, and for those orbits we used the local fitting and conformal fitting methods to calculate the tilt.

We find that the tilt term depends primarily on the $z$ velocity of a star and hence the height to which it reaches above the galactic plane, and very weakly on either the $R$ or $\phi$ velocities. The dependence on the latter two quantities is further reduced when averaging over a distribution of velocities. Hence to calculate the tilt term, it is not necessary to have an accurate distribution function for the stars. The ensemble-averaged $\langle C\rangle$ is a function principally of the vertical velocity dispersion $\sigma_{z z}$.

For the old disk stars in the solar neighborhood, we have approximated the distribution function of velocities by a Gaussian in each direction with $\sigma_{R R}=40 \mathrm{~km} \mathrm{~s}^{-1}, \sigma_{\phi \phi}=25$ $\mathrm{km} \mathrm{s}^{-1}$, and $\sigma_{z z}=20 \mathrm{~km} \mathrm{~s}^{-1}$ (e.g., Freeman 1987). We find that the correction factor varies from 0.49 for stars with very low $z$ velocities to about 0.66 for stars that pass through the solar neighborhood with a $z$ velocity of $100 \mathrm{~km} \mathrm{~s}^{-1}$, roughly according to the law $C=0.49+0.017 \sqrt{V_{z}}$. (Note that the local fitting and conformal approximations both predict a value of about 0.49 for all orbits.) The mean correction factor $\langle C\rangle$ for the entire ensemble of orbits is about 0.51 .

Kuijken \& Gilmore (1989a) point out that for stars with low angular momentum, new families of orbits around higher-order resonances appear. Our third integral approximations cannot properly describe such orbits. However, in our survey of orbits for stars in the solar neighborhood, we found that only stars of extreme velocities follow those types of orbits, and so we would expect that our computation of $\langle C\rangle$ is still valid.

We have also evaluated the variation in $\langle C\rangle$ with both 
height above the plane and with radius. For this purpose, it is necessary to know the variation in $\sigma_{z z}$ with $R$ and $z$. Following Kuijken \& Gilmore (1989a), we assume that $\sigma_{z z}^{2} \propto \exp (-R / H)$, where $H=4.5 \mathrm{kpc}$ is the disk exponential scale length. In the vertical direction we find that $d\langle C\rangle / d z=0.15 \mathrm{kpc}^{-1}$ up to a height of $600 \mathrm{pc}$, at which point the small-angle approximation in Eq. (33) starts breaking down. The reason that $\langle C\rangle$ increases is that stars at high $z$ can reach still farther on average from the galactic plane than stars in the solar neighborhood and hence their orbits on average have larger values of $C$. In the radial direction, we find $d\langle C\rangle / d R=0.05 \mathrm{kpc}^{-1}$.

In their derivation of the Galactic disk surface mass density in the solar neighborhood, Kuijken \& Gilmore (1989b) found that their value of the mass density increases from 41 to $50 \mathscr{M}_{\odot} \mathrm{pc}^{-2}$ as $\langle C\rangle$ increases from 0 to 1 . Now the stars used in their analysis have $z$ heights that range from 300 to over $2 \mathrm{kpc}$, so strictly speaking we cannot interpolate to a single value of $\langle C\rangle$; however, the median height of their stars is about $600 \mathrm{pc}$, where we find $\langle C\rangle=0.60$ and hence we would select a surface density of $46 \mathscr{M}_{\odot} \mathrm{pc}^{-2}$.

Our calculation of the tilt term is subject to several "errors" which are difficult to quantify. The biggest one is that we have restricted ourselves to the Carlberg-Innanen potential of the Galaxy, and it would be interesting (although we have not yet done so) to see how it varies as one makes plausible variations in the details of the Galactic mass model. We have used only a rough distribution function for the stellar velocities, but we stress that complete knowledge of the distribution function is not necessary and in practice only the velocity dispersion in the $Z$ direction is important. The computation of $\langle C\rangle$ is also complicated in principle by the presence of higher-order resonant orbits for which our third integral approximations break down, but at least in the Carlberg and Innanen potential, such orbits are unimportant for the old disk stars.

\section{CONCLUSIONS}

We have developed three methods for approximating the orbits of stars in axisymmetric potentials. All methods approximate the orbits by motion in a separable potential. The least-squares method, which tailors a spheroidal coordinate system and approximating potential to each orbit, gives the most accurate results and is fairly straightforward to implement. The local-fitting method, which uses a single coordinate system and approximating potential for all orbits of the same $h$ but different $E$ and $I_{3}$, is quite simple to implement but is not as accurate as the least-squares method. The conformal fitting method is complicated to implement and is probably of interest only for sufficiently complicated potentials where the Stäckel approximations break down.

These methods have been used to compute the tilt of the velocity ellipsoid for disk stars in the solar neighborhood based on a specific model for the Galactic potential. For small distances above the Galactic plane, we find that the tilt term can be written in the form

$$
\sigma_{R z}^{2}=\langle C\rangle\left(\sigma_{R R}^{2}-\sigma_{z z}^{2}\right) z / R,
$$

where $\langle C\rangle$ is a correction factor to the value that this term would have if the velocity ellipsoid pointed towards the $\mathrm{Ga}$ lactic center. For old disk stars, we find that

$$
\langle C\rangle \approx 0.51+0.15 z_{\mathrm{kpc}}+0.05 R_{\mathrm{kpc}}
$$

over the range $6.8<R<8.8 \mathrm{kpc}$ and $0<z<600$ pc. Al- though the tilt term has often been treated as an unknown in dynamical problems that use the equations of hydrostatic equilibrium, we find that it can be calculated to good precision without the need to have a complete three-integral model for the stellar distribution function. Compared with other terms in the equations of hydrostatic equilibrium, however, the tilt term is special in that it is the only one that depends on the global Galactic potential (or at least in a region of a few kpc about the solar neighborhood). In Kuijken \& Gilmore's $(1989 a, b)$ problem of estimating the disk mass density, the Galactic potential is modeled as part of the problem, so in principle one could demand that the tilt term calculation be self-consistent with the model potential.

McGill \& Binney (1990) developed a least-squares fitting method for approximating orbits based on action integrals. Their method is analogous in spirit to our least-squares fitting method and is even more general. In local fitting, and also in our version of least-squares fitting, the actions can be found easily by evaluating the appropriate expressions in the Stäckel potential that best fits the orbit. For the problem of computing the tilt of the velocity ellipsoid in the solar neighborhood, the orbits involved are sufficiently simple that the added complication of working directly with the action integrals is probably unnecessary.

This work was supported by NSF Grant No. AST 8451724 and the Digital Equipment Corporation. Discussions with S. Tremaine in an earlier stage of this work are gratefully acknowledged.

\section{APPENDIX: SPHEROIDAL COORDINATES}

There are a variety of different notations and conventions for prolate spheroidal coordinates. The conformal definition used in this paper is identical to the one employed by Binney \& Tremaine (1987). Here we compare it with two other popular conventions.

Morse \& Feshbach (1953) denote prolate spheroidal coordinates by $(\xi, \phi, \eta)$, where $\xi$ and $\eta$ are related to $u$ and $v$ by

$$
\xi=\cosh u, \quad \eta=\sin v \text {. }
$$

These authors use $D$, rather than $\Delta$, as semifocal distance.

In many recent papers on oblate Stäckel models (e.g., de Zeeuw \& Hunter 1990, and references therein), prolate spheroidal coordinates of a point $(R, \phi, z)$ are defined, following Jacobi (1866), by replacing $R$ and $z$ by the two roots for $\tau$ of

$$
\frac{R^{2}}{\tau+\alpha}+\frac{z^{2}}{\tau+\gamma}=1,
$$

where $\alpha$ and $\gamma$ are constants. The roots are usually denoted $\lambda$ and $v$, and they are chosen such that $-\gamma \leqslant v \leqslant-\alpha \leqslant \lambda<\infty$. The foci lie on the $z$ axis, at $z= \pm \sqrt{\gamma-\alpha}$. It follows that

$$
\begin{aligned}
& \lambda+\gamma=\Delta^{2} \cosh ^{2} u, \\
& v+\gamma=\Delta^{2} \sin ^{2} v,
\end{aligned}
$$

where

$$
\Delta^{2}=\gamma-\alpha
$$

As a result

$$
\begin{aligned}
& \lambda+\alpha=\Delta^{2} \sinh ^{2} u, \\
& v+\alpha=-\Delta^{2} \cos ^{2} v,
\end{aligned}
$$$$
\text { and }
$$$$
\lambda-v=\Delta^{2}\left(\cosh ^{2} u-\sin ^{2} v\right)=\Delta^{2}\left(\sinh ^{2} u+\cos ^{2} v\right) .
$$ 
REFERENCES

Binney, J. J., and Tremaine, S. D. 1987, Galactic Dynamics (Princeton University Press, Princeton, NJ)

Carlberg, R. G., and Innanen, K. A. 1987, AJ, 94, 666

Contopoulos G. 1960, Zeits. Astrophys., 49, 273

de Zeeuw, P. T. 1984, Ph.D. thesis, Leiden University, Chap. 7

de Zeeuw, P. T. 1985, MNRAS, 216, 273

de Zeeuw, P. T., and Lynden-Bell, D. 1985, MNRAS, 215, 713

de Zeeuw, P. T., and Hunter, C. 1990, ApJ, 356, 365

Eddington, A. S. 1915, MNRAS, 76, 37

Freeman, K. C. 1987, ARA\&A, 25, 603

Hietarinta, J. 1987, Phys. Rep., 147, 87

Hori, G. 1962, PASJ, 14, 353

Jacobi, C. G. J. 1866, Vorlesungen über Dynamik (Reimer, Berlin)

Jeans, J. H. 1915, MNRAS, 76, 71

Kuijken, K., and Gilmore, G. 1989a, MNRAS, 239, 571

Kuijken, K., and Gilmore, G. 1989b, MNRAS, 239, 605
Kuzmin, G. G. 1956, AZh, 33, 27

Lynden-Bell, D. 1962, MNRAS, 124, 95

Manabe, S. 1979, PASJ, 31, 369

Martinet, L. 1974, A\&A, 32, 329

McGill, C., and Binney, J. J. 1990, MNRAS, 244, 634

Morse, P. M., and Feshbach, H. 1953, Methods of Theoretical Physics (McGraw-Hill, New York), Chap. 5

Ollongren, A. 1962, Bull. Astr. Inst. Netherlands, 16, 241

Oort, J. H. 1928, Bull. Astr. Inst. Netherlands, 4, 269

Press, W. H., Flannery, B. P., Teukolsky, S. A., and Vetterling, W. T. 1986,

Numerical Recipes (Cambridge University Press, Cambridge)

Saaf, A. F. 1968, ApJ, 154, 483

Stäckel, P. 1890, Math. Ann., 42, 537

Statler, T. 1989, ApJ, 344, 217

van de Hulst, H. C. 1962, Bull. Astr. Inst. Netherlands, 16, 235

Verhulst, F. 1979, Philos. Trans. R. Soc. London, 290, 435 